\title{
A novel formulation of 3D spectral element for wave propagation in reinforced concrete
}

\author{
M. RUCKA*, W. WITKOWSKI, J. CHRÓŚCIELEWSKI, S. BURZYŃSKI, and K. WILDE \\ Faculty of Civil and Environmental Engineering, Gdańsk University of Technology, 11/12 Narutowicza St., 80-233 Gdańsk, Poland
}

\begin{abstract}
The paper deals with numerical simulations of wave propagation in reinforced concrete for damage detection purposes. A novel formulation of a 3D spectral element was proposed. The reinforcement modelled as the truss spectral element was embedded in the 3D solid spectral finite element. Numerical simulations have been conducted on cuboid concrete specimens reinforced with two steel bars. Different degradation models were considered to study the real behaviour of bended beams.
\end{abstract}

Key words: spectral element method, reinforced concrete, wave propagation, degradation.

\section{Introduction}

Reinforced concrete is commonly used for construction of civil infrastructure including buildings, towers, bridges, silos or dams. Continuous environmental and operational loadings cause degradation of material parameters of concrete. The appearance of cracks in reinforced concrete structures is a natural process and zones of micro-cracks or small cracks of permissible size do not affect the durability of concrete elements. However, large, open cracks can cause a failure of an entire structure. Therefore, damage detection and monitoring of damage development is of great interest in civil engineering. Recently, various non-destructive testing (NDT) methods have been developed for evaluation of concrete structures, e.g. vibration-based methods [1], ground penetrating radar [2], acoustic emission [3], or infrared thermography [4]. Methods utilizing the phenomenon of wave propagation, including ultrasonic testing, the impact-echo method and the Lamb wave modes [5-9] are particularly interesting. The advantage of wave propagation-based methods is that they may be used in automatic structural health monitoring (SHM) systems. It has been demonstrated that wave propagation methods can be efficiently applied in monitoring of the strain changes in reinforced concrete during the process of damage development [10-13]. Wave propagation signals collected during an experiment conducted on concrete structures are characterized by a greater degree of complexity than for steel thin rods [14] or plates [15] because of the solid geometry of typical concrete element. Damage detection methodologies utilizing wave propagation signals directed to concrete structures require a lot of testing data for different stages of damage. Since experiments are very expensive and time-consuming, numerical simulations can enhance the understanding of wave propagating in reinforced concrete.

*e-mail: mrucka@pg.edu.pl

Manuscript submitted 2017-01-03, revised 2017-04-28, initially accepted for publication 2017-05-17, published in December 2017.
In wave propagation modelling the spectral element method (SEM) plays an important role [16]. The SEM method is based on the high-order finite elements, usually of class $C^{0}$. As a special feature, the elements have their nodes distributed both in physical space and in parent elements according to the Gauss-Lobatto-Legendre quadrature rule. As a consequence, it is possible to obtain a diagonal mass matrix at the element level which in turn, when appropriately taken into account, leads to fast and efficient time marching scheme. A multitude of aspects pertaining to SEM, especially for modelling of seismic waves (e.g. $[17,18]$ ) as well as for wave propagation for SHM purposes (e.g. [19]), have been already discussed. Spectral elements have been also developed for structural type finite elements like rods, beams or plates (e.g. [14, 15, 20-27]), with the attention paid to theoretical aspects of wave propagation phenomena. In paper [28], 3-dimensional spectral elements have been presented, with application to damage detection in metal plate structures. Unbounded structures need a special treatment to preserve the dissipation of the energy in the infinite. The use of absorbing layers has been proposed in [29], on the example of a 1-dimensional rod and a 3-dimensional half-cylindrical shell. As far as damage detection is concerned, various techniques applied to simulation of damage effects have been considered. One can refer for instance to [20-22] where discontinuities in the form of cracks/caverns or point masses have been accounted for. A semi-analytical approach in analysis of flexural wave propagation through a slender beam, with a breathing edge-crack, is developed in [30]. In the context of non-homogenous material reference [31] discusses the use of the SEM with functionally graded material (FGM). To the authors' best knowledge, the problem of wave propagation in damaged reinforced concrete with the use of SEM has been not investigated.

The present study focuses on development of a novel 3D spectral element for wave propagation in reinforced concrete subjected to mechanical degradation. Numerical simulations have been conducted on cuboid concrete specimens reinforced with two steel bars. Different degradation models were considered to reflect the real behaviour of bended beams described in [11]. 


\section{Formulation of 3D spectral elements with embedded 1D truss elements}

2.1 Initial-boundary value problem of linear elasticity. To fix the ideas of the present approach we briefly summarise the necessary ingredients of linear elasticity in the notation pertaining to the FEM formulation. The presentation is provided for 3-dimensional formulation, since 1-dimensional follows immediately. The details are to be found in classical text books, for instance, [32, 33].

We assume a fixed 3-dimensional Cartesian reference system with base vectors $\mathbf{e}_{\mathrm{i}}, i=1,2,3$. Consider an elastic body $B$ of which particles are determined by the position vector $\mathbf{x}=\left\{x_{1}, x_{2}, x_{3}\right\}^{T}$ under action of time prescribed body force $\mathbf{f}$ and displacement $\mathbf{u}(t)$ :

$$
\mathbf{f}=\left\{\begin{array}{l}
f_{1} \\
f_{2} \\
f_{3}
\end{array}\right\} \text { on } B, \quad \mathbf{u}=\left\{\begin{array}{ll}
u_{1}(t) \\
u_{2}(t) \\
u_{3}(t)
\end{array}\right\} \text { on } B, \quad \begin{aligned}
& \mathbf{v}(t)=\dot{\mathbf{u}}(t), \\
& \mathbf{a}(t)=\ddot{\mathbf{u}}(t),
\end{aligned}
$$

where $t \in[0, T]$ stands for time and superposed dot denotes time derivative in linear elasticity. As usual, we specify displacement boundary conditions $\overline{\mathbf{u}}$ and traction boundary conditions $\mathbf{t}^{*}$ on respective parts of the boundary $\partial B=\partial B_{d} \cup \partial B_{f}$ assuming that $\partial B_{d} \cap \partial B_{f}=\varnothing:$

$$
\overline{\mathbf{u}}=\left\{\begin{array}{l}
\bar{u}_{1} \\
\bar{u}_{2} \\
\bar{u}_{3}
\end{array}\right\} \text { along } B_{d}, \quad \mathbf{t}^{*}=\left\{\begin{array}{l}
t_{1}^{*} \\
t_{2}^{*} \\
t_{3}^{*}
\end{array}\right\} \text { along } \partial B_{f} .
$$

In the infinitesimal case the strain $\boldsymbol{\varepsilon}(t)$ is defined as the (symmetric) gradient of $\mathbf{u}(t)$ :

$$
\boldsymbol{\varepsilon}(t)=\left\{\begin{array}{c}
\varepsilon_{11} \\
\varepsilon_{22} \\
\varepsilon_{33} \\
2 \varepsilon_{23} \\
2 \varepsilon_{31} \\
2 \varepsilon_{12}
\end{array}\right\}=\mathbf{D u}, \quad \mathbf{D}=\left[\begin{array}{ccc}
\frac{\partial}{\partial x} & 0 & 0 \\
0 & \frac{\partial}{\partial y} & 0 \\
0 & 0 & \frac{\partial}{\partial z} \\
0 & \frac{\partial}{\partial z} & \frac{\partial}{\partial y} \\
\frac{\partial}{\partial z} & 0 & \frac{\partial}{\partial x} \\
\frac{\partial}{\partial y} & \frac{\partial}{\partial x} & 0
\end{array}\right] .
$$

In the case of linear elastic homogenous material, the constitutive equation reads

$$
\boldsymbol{\sigma}=\mathbf{E} \boldsymbol{\varepsilon},
$$

where $\boldsymbol{\sigma}$ is the stress and $\mathbf{E}$ denotes the constitutive matrix. The explicit form of (4) will be discussed later. Assuming the initial conditions $\mathbf{u}(0)=\mathbf{u}_{0}, \dot{\mathbf{u}}(0)=\mathbf{v}_{0}$ the weak form of IBV problem may be stated as: given $\mathbf{f}, \mathbf{t}^{*}, \mathbf{u}_{0}, \mathbf{v}_{0}$, find kinematically admissible $\mathbf{u}(t) \in U, t \in[0, T]$ such that $\forall \mathbf{w}$

$$
\begin{aligned}
G[\mathbf{u} ; \delta \mathbf{u}] & =\int_{B}(\mathbf{D} \delta \mathbf{u})^{\mathrm{T}} \mathbf{E}(\mathbf{D u}) \mathrm{d} V+\int_{B} \rho(\delta \mathbf{u})^{\mathrm{T}} \ddot{\mathbf{u}} \mathrm{d}+ \\
& -\left(\int_{B} \delta \mathbf{u}^{\mathrm{T}} \mathbf{f} \mathrm{d} V+\int_{\partial B_{f}} \delta \mathbf{u}^{\mathrm{T}} \mathbf{t}^{*} \mathrm{~d} A\right)=0 .
\end{aligned}
$$

For equation (5) to make sense it is necessary that the admissible virtual displacements $\mathbf{w}$ satisfy homogenous boundary conditions $\mathbf{w}(\mathbf{x})=\mathbf{0}, \mathbf{x} \in \partial B_{d}$ and the functions $\mathbf{u}(\mathbf{x})=\overline{\mathbf{u}}, \mathbf{x} \in \partial B_{d}$ and $\mathbf{w}(\mathbf{x})$ satisfy some regularity conditions.

2.2. Spatial and temporal approximation. Assume that the domain of discretization $B$ is approximated by non-overlapping finite elements $B_{(\mathrm{e})}$ i.e.

$$
\bigcup_{\mathrm{e}=1}^{M}\left(B_{(\mathrm{e})} \cup \partial B_{(\mathrm{e})}\right)=\left\{\begin{array}{l}
B \cup \partial B \\
B_{h} \cup \partial B_{h} \rightarrow B \cup \partial B \text { if } h \rightarrow \infty
\end{array}\right.
$$

with $M$ being the number of elements. We introduce the following interpolation $\mathbf{u} \rightarrow \tilde{\mathbf{u}}$ within the $N$-th element:

$$
\begin{aligned}
& \tilde{\mathbf{u}}(\mathbf{x})=\sum_{a=1}^{N_{(\mathrm{e})}} \mathbf{N}_{a}(\mathbf{x}) \mathbf{u}_{a}=\mathbf{N}_{(\mathrm{e})}(\mathbf{x}) \mathbf{u}_{(\mathrm{e})}, \\
& \mathbf{x}(\boldsymbol{\xi}) \in B_{(\mathrm{e})} \cup \partial B_{(\mathrm{e})},
\end{aligned}
$$

where $N_{(\mathrm{e})}$ is the number of nodes, $\xi=\pi_{(\mathrm{e})} \cup \partial \pi_{(\mathrm{e})}, \boldsymbol{\xi}=\left(\xi^{1}, \xi^{2}, \xi^{3}\right)$ $\in[-1,+1] \times[-1,+1] \times[-1,+1] \subset R^{3}$ denotes the coordinates of the parent element and:

$$
\begin{gathered}
\mathbf{N}_{(\mathrm{e})}(\boldsymbol{\xi})=\left[\mathbf{N}_{1}(\boldsymbol{\xi}) \mathbf{N}_{2}(\boldsymbol{\xi}) \ldots \mathbf{N}_{a}(\boldsymbol{\xi}) \ldots \mathbf{N}_{N_{(\mathrm{e})}}(\boldsymbol{\xi})\right], \\
\mathbf{N}_{a}(\boldsymbol{\xi})=\left[\begin{array}{ccc}
N_{a}^{x}(\boldsymbol{\xi}) & 0 & 0 \\
0 & N_{a}^{y}(\boldsymbol{\xi}) & 0 \\
0 & 0 & N_{a}^{z}(\boldsymbol{\xi})
\end{array}\right], \\
\mathbf{u}_{(\mathrm{e})}=\left\{\begin{array}{c}
\mathbf{u}_{1} \\
\vdots \\
\mathbf{u}_{a} \\
\vdots \\
\mathbf{u}_{N_{(\mathrm{e})}}
\end{array}\right\}, \quad \mathbf{u}_{a}=\left\{\begin{array}{l}
u_{a 1} \\
u_{a 2} \\
u_{a 3}
\end{array}\right\} \equiv\left\{\begin{array}{c}
u_{1} \\
u_{2} \\
u_{3}
\end{array}\right\} \mid,
\end{gathered}
$$

are the element and nodal arrays of shape functions and nodal parameters, respectively. In (8) the upper labels $x, y, z$ signify that different orders of Lagrange polynomials may be used in each spatial dimension $x, y, z$. It is worthy of note that the possibility of selecting of different orders of approximation in 
a)

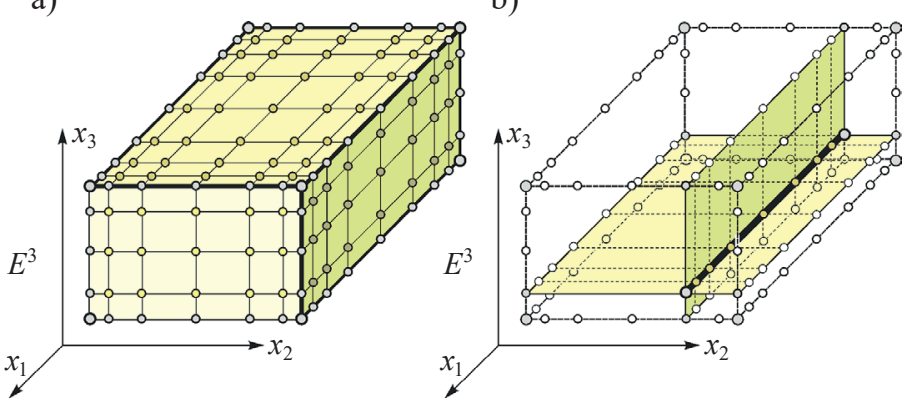

Fig. 1. a) 3D spectral finite element, b) spectral truss element (in black) embedded in the $3 \mathrm{D}$ spectral element

different spatial directions is important and should be always properly chosen for a given problem at hand. The presented formulation makes it possible to use different numbers on nodes in each spatial direction, see Fig. 1.

With these definitions the (continuous) weak formulation equation (5) is approximated as

$$
\begin{aligned}
& G\left[\mathbf{u}_{h} ; \delta \mathbf{u}_{h}\right]= \\
& =\sum_{\mathrm{e}=1}^{M}\left(\begin{array}{l}
\int_{B_{(\mathrm{e})}}(\mathbf{D} \delta \tilde{\mathbf{u}})^{\mathrm{T}} \mathbf{E}(\mathbf{D} \tilde{\mathbf{u}}) \mathrm{d} V+ \\
+\int_{B_{(\mathrm{e})}} \rho(\delta \tilde{\mathbf{u}})^{\mathrm{T}} \ddot{\tilde{\mathbf{u}}} \mathrm{d} V+ \\
-\left(\int_{B_{(\mathrm{e})}} \delta \tilde{\mathbf{u}}^{\mathrm{T}} \mathbf{f} \mathrm{d} V+\int_{\partial B_{(\mathrm{e})} \cap \partial B_{f}} \delta \tilde{\mathbf{u}}^{\mathrm{T}} \mathbf{t}^{*} \mathrm{~d} A\right)
\end{array}\right)=0 .
\end{aligned}
$$

Introducing strain-displacement operator $\mathbf{B}_{(\mathrm{e})}=\mathbf{D N}_{(\mathrm{e})}$ yields

$$
G\left[\mathbf{u}_{h} ; \delta \mathbf{u}_{h}\right]=\sum_{\mathrm{e}=1}^{M} \delta \mathbf{u}_{(\mathrm{e})}^{\mathrm{T}}\left\{\mathbf{K}_{(\mathrm{e})} \mathbf{u}_{(\mathrm{e})}+\mathbf{M}_{(\mathrm{e})} \ddot{\mathbf{u}}_{(\mathrm{e})}-\mathbf{p}_{(\mathrm{e})}\right\},
$$

with standard definitions of the stiffness matrix $\mathbf{K}_{(\mathrm{e})}$, mass ma$\operatorname{trix} \mathbf{M}_{(\mathrm{e})}$ :

$$
\mathbf{K}_{(\mathrm{e})}=\int_{B_{(\mathrm{e})}} \mathbf{B}_{(\mathrm{e})}^{\mathrm{T}} \mathbf{E} \mathbf{B}_{(\mathrm{e})} \mathrm{d} V, \quad \mathbf{M}_{(\mathrm{e})}=\int_{B_{(\mathrm{e})}} \rho \mathbf{N}_{(\mathrm{e})}^{\mathrm{T}} \mathbf{N}_{(\mathrm{e})} \mathrm{d} V,
$$

and the element load vector $\mathbf{p}_{(\mathrm{e})}$ :

$$
\mathbf{p}_{(\mathrm{e})}=\int_{B_{(\mathrm{e})}} \mathbf{N}_{(\mathrm{e})}^{\mathrm{T}} \mathbf{f d} V+\int_{\partial B_{(\mathrm{e})} \cap \partial B_{f}} \mathbf{N}_{(\mathrm{e})}^{\mathrm{T}} \mathbf{t}^{*} \mathrm{~d} A
$$

For the purposes of temporal discretization, cf. also [21, $22,33,34]$, we define at the element level the vector of inertia forces $\mathbf{b}_{(\mathrm{e})}$ and internal forces $\mathbf{r}_{(\mathrm{e})}$ i.e.:

$$
\mathbf{b}_{(\mathrm{e})}=\mathbf{M}_{(\mathrm{e})} \ddot{\mathbf{u}}_{(\mathrm{e})}, \quad \mathbf{r}_{(\mathrm{e})}=\int_{B_{(\mathrm{e})}} \mathbf{B}_{(\mathrm{e})}^{\mathrm{T}}(\boldsymbol{\xi}) \boldsymbol{\sigma}_{(\mathrm{e})}(\boldsymbol{\xi}) \mathrm{d} V
$$

As a result of aggregation procedure global equation of motion is obtained as

$$
\mathbf{M} \ddot{\mathbf{q}}+\mathbf{C} \dot{\mathbf{q}}=\mathbf{p}-\mathbf{r}(\mathbf{q})
$$

where $\mathbf{q}, \ddot{\mathbf{q}}$ and $\dot{\mathbf{q}}$ are structural vectors of displacements, accelerations and velocities, respectively. The damping matrix $\mathbf{C}$ and damping forces $\mathbf{c}$ are defined as:

$$
\mathbf{C}=\eta \mathbf{M}, \quad \mathbf{c}=\mathbf{C} \dot{\mathbf{q}}
$$

In (16) we do not use make the damping matrix dependent on the stiffness matrix (12) $)_{1}$ since the latter is not diagonal. The shape functions $N_{a}^{x, y, z}(\xi)$ in (8) are defined as the product $N_{a}(\boldsymbol{\xi})=L_{b}^{p}\left(\xi^{1}\right) L_{c}^{q}\left(\xi^{2}\right) L_{d}^{r}\left(\xi^{3}\right),\left(\xi^{1}, \xi^{2}, \xi^{3}\right) \in[-1,+1] \times[-1,+1] \times$ $\times[-1,+1]$ of Lagrange polynomials:

$$
L_{p}^{m}(\xi)=\prod_{q \neq p, q}^{m} \frac{\xi-\xi_{(q)}}{\xi_{(p)}-\xi_{(q)}} .
$$

As the interpolation nodes in the parent and physical element, we select nodes placed according to the Gauss-Lobatto-Legendre quadrature i.e.

$$
\left(1-\xi^{2}\right) \frac{d P^{M-1}(\xi)}{d \xi}=0, \quad \xi \leftarrow \xi^{1}, \xi^{2}, \xi^{3},
$$

with $P^{M}$ being the Legendre polynomial. A typical 3D spectral element with embedded rod is shown in Fig. 1. The placement of the internal nodes in the physical space maps the placement of the nodes in the parent element.

As a consequence of using the GLL nodes and with definition (12) the mass matrix and damping matrix (16) become diagonal at element level and at the global level in (15). This renders profound speed-up when solving equation of motion (15) as discussed in [21, 22, 33, 34]. Briefly, the employed time integration scheme is based on the following relations:

$$
\ddot{\mathbf{q}}_{n+1}^{(i+1)}=\ddot{\mathbf{q}}_{n+1}^{(i)}+\delta \ddot{\mathbf{q}}
$$

$$
\begin{aligned}
\dot{\mathbf{q}}_{n+1}^{(i+1)} & =\underbrace{\dot{\mathbf{q}}_{n}+\Delta t\left[(1-\gamma) \ddot{\mathbf{q}}_{n}+\gamma \ddot{\mathbf{q}}_{n+1}^{(i)}\right]}_{\dot{\mathbf{q}}_{n+1}^{(i)}}+ \\
& +\Delta t \gamma \delta \ddot{\mathbf{q}}=\dot{\mathbf{q}}_{n+1}^{(i)}+\Delta t \gamma \delta \ddot{\mathbf{q}},
\end{aligned}
$$

$$
\begin{aligned}
\mathbf{q}_{n+1}^{(i+1)} & =\underbrace{\mathbf{q}_{n}+\Delta t \dot{\mathbf{q}}_{n}+\frac{1}{2}(\Delta t)^{2}\left[(1-2 \beta) \ddot{\mathbf{q}}_{n}+2 \beta \ddot{\mathbf{q}}_{n+1}^{(i)}\right]}_{\ddot{\mathbf{q}}_{n+1}^{(i)}}+ \\
& +(\Delta t)^{2} \beta \delta \ddot{\mathbf{q}}=\ddot{\mathbf{q}}_{n+1}^{(i)}+(\Delta t)^{2} \beta \delta \ddot{\mathbf{q}},
\end{aligned}
$$

where $\gamma$ and $\beta$ are defined as for the average acceleration method i.e. $\gamma=1 / 2, \beta=1 / 4$. Here $n$ denotes time instance $\Delta t=t_{n}-t_{n-1}$ and $i$ is the iteration counter. Substitution of (21) into (15) results in implicit relation for $\delta \ddot{\mathbf{q}}$ i.e.

$$
\begin{aligned}
{[\mathbf{M}+\Delta t \gamma \mathbf{C}] \delta \ddot{\mathbf{q}} } & =\mathbf{p}_{n+1}-\mathbf{b}_{n+1}^{(i)}-\mathbf{c}_{n+1}^{(i)}- \\
& +\mathbf{r}\left(\mathbf{q}_{n+1}^{(i)}+(\Delta t)^{2} \beta \delta \ddot{\mathbf{q}}\right)
\end{aligned}
$$


which is solved using simple iteration yielding

$$
\delta \ddot{\mathbf{q}}=[\mathbf{M}+\Delta t \gamma \mathbf{C}]^{-1}\left(\mathbf{p}_{n+1}-\mathbf{b}_{n+1}^{(i)}-\mathbf{c}_{n+1}^{(i)}-\mathbf{r}\left(\mathbf{q}_{n+1}^{(i)}\right)\right),
$$

Equation (23) is solved until an equilibrium condition is satisfied

$$
\mathbf{j}_{n+1}^{(i+1)}=\mathbf{p}_{n+1}-\mathbf{b}_{n+1}^{(i+1)}-\mathbf{c}_{n+1}^{(i+1)}-\mathbf{r}\left(\mathbf{q}_{n+1}^{(i+1)}\right) \rightarrow \mathbf{0},
$$

The iterations are terminated if the (relative) convergence criteria are satisfied

$$
\begin{aligned}
\max _{k} \frac{\left|\delta \ddot{q}_{k}\right|}{\left|\ddot{q}_{k n+1}^{(i+1)}-\ddot{q}_{k n}\right|}<\varepsilon_{a}, \quad \frac{\|\delta \ddot{\mathbf{q}}\|}{\left\|\ddot{\mathbf{q}}_{n+1}^{(i+1)}-\ddot{\mathbf{q}}_{n}\right\|}<\varepsilon_{b}, \\
0<\varepsilon_{a}, \varepsilon_{b} \ll 1,
\end{aligned}
$$

where $\|$.$\| is Euclidean norm of a vector i.e.$

$$
\|\boldsymbol{a}\|=\left(\sum_{i=1}^{3} a_{i}^{2}\right)^{1 / 2} .
$$

2.3. Constitutive equation, degradation models. In the present formulation, the integration points and the element nodes coincide. It is therefore relatively easy to define the properties of the elastic isotropic material that varies spatially within the volume of a structure. In this sense, the approach presented here has close connection with the functionally graded materials $[35,36]$. That said we select some regions where we assign (pointwise i.e. at the FEM nodes) different values of the Young's modulus $E$ in Eq. (27), leaving unchanged values of the Poisson's ratio $v$ and the density. In other words we assume that Eq. (4) is dependent on the spatial coordinate $\mathbf{x}$ in such a way that:

$$
\boldsymbol{\sigma}(\mathbf{x})=\mathbf{E}(E(\mathbf{x}), v) \boldsymbol{\varepsilon}(\mathbf{x})
$$

Assuming the linear elastic isotropic homogenous material we have the following constitutive matrix:

$$
\mathbf{E}(E(\mathbf{x}), v)=\frac{E(\mathbf{x})}{(1+v)(1-2 v)} \mathbf{A},
$$

where $E$ is the Young's modulus, $v$ denotes the Poisson's ratio and $\mathbf{A}$ is:

$$
\mathbf{A}=\left[\begin{array}{cccccc}
1-v & v & v & 0 & 0 & 0 \\
v & 1-v & v & 0 & 0 & 0 \\
v & v & 1-v & 0 & 0 & 0 \\
0 & 0 & 0 & \frac{1}{2}(1-2 v) & 0 & 0 \\
0 & 0 & 0 & 0 & \frac{1}{2}(1-2 v) & 0 \\
0 & 0 & 0 & 0 & 0 & \frac{1}{2}(1-2 v)
\end{array}\right] .
$$

This is by no means a simplification. Yet it can be supported by the following argument. If the ultrasonic wave propagation is to be employed in the non-destructive evaluation, it is usual that we do not observe progressive damage taking place in the time instance of performing the measurement. Rather, we deal with destroyed structure, in which we observe the results of damage in the form of microcracks, holes, caverns, etc.

\section{Numerical analyses of wave propagation in reinforced concrete}

The FEM analysis was conducted using own programme written on Fortran, according to the algorithm described in the previous section. As an example, a reinforced concrete specimen was chosen, on the basis of the previous experimental results presented in paper [11]. Therefore, it was possible to compare numerical results with experimental ones.

3.1. Description of specimen. The analysed structure was a rectangular prism with a cross-section of $10 \mathrm{~cm} \times 15 \mathrm{~cm}$ and a length of $50 \mathrm{~cm}$ (Fig. 2). The material was assumed as concrete with modulus of elasticity $E=30 \mathrm{GPa}$, density $\rho=2300 \mathrm{~kg} / \mathrm{m}^{3}$ and Poisson's ratio $v=0.16$, damping parameter $\eta=3000$. The specimen was reinforced in the longitudinal direction with two steel bars of a $10 \mathrm{~mm}$ diameter with the following material parameters: $E=205 \mathrm{GPa}, \rho=7850 \mathrm{~kg} / \mathrm{m}^{3}, v=0.3$.

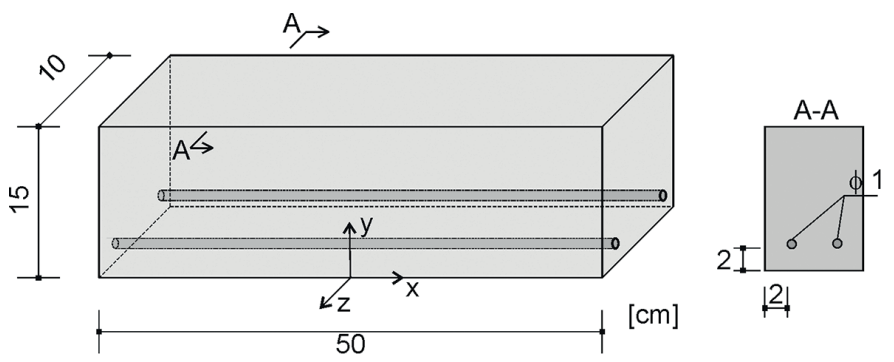

Fig. 2. Geometry and dimensions of reinforced concrete specimen

3.2. Numerical model. The numerical model consisted of $3 \mathrm{D}$ spectral elements with $7 \times 7 \times 7$ GLL nodes. The reinforced concrete specimen was divided into 3840 elements: 40 elements in the length direction, 12 elements in the height direction and 8 elements in the width direction. The reinforcement bars were modelled with 1D spectral elements with 7 GLL nodes. Each of the bars was meshed into 40 elements in the length direction. The equation of motion was integrated using time step $\Delta t=1 \times 10^{-7} \mathrm{~s}$. In the calculations performed in this paper one iteration was necessary to satisfy the criteria given by Eq. (25) assumed as $\varepsilon_{a}=\varepsilon_{b}=10^{-7}$.

3.3. Degradation models. Crack pattern of the RC specimen considered in this study was adopted based on experiments of three-point bending [11]. In the study described in [11] the specimen was subjected to mechanical degradation process 
a)

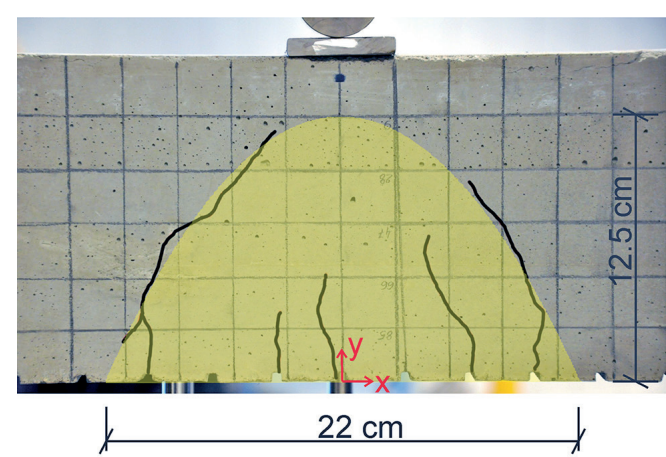

b)

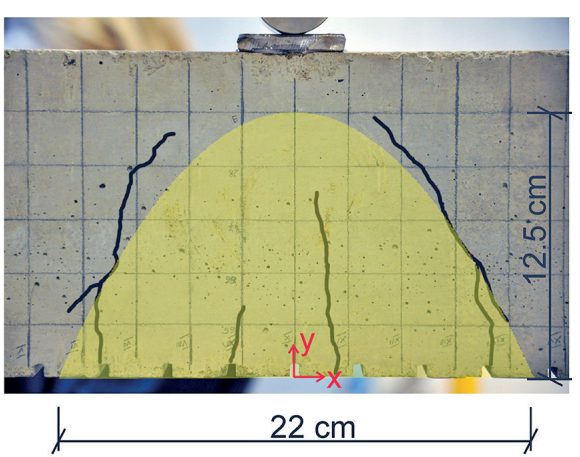

Fig. 3. Crack patterns for reinforced concrete specimens subjected to mechanical degradation [11] and approximation of cracked zone with parabolic area: a) specimen $\# 1$; b) specimen $\# 2$

(a)

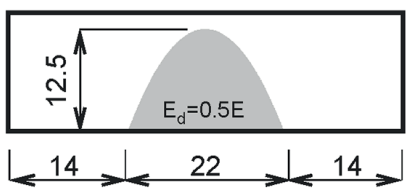

(c)

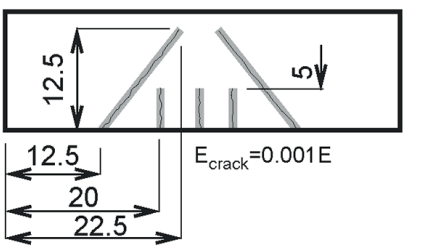

(b)

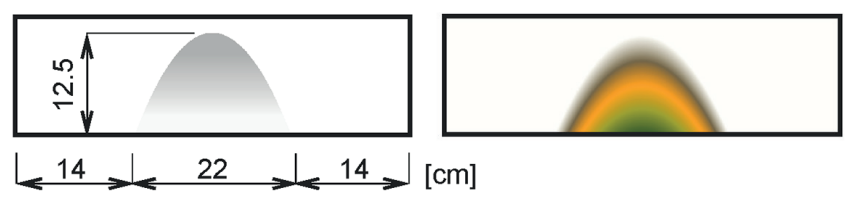

(d)

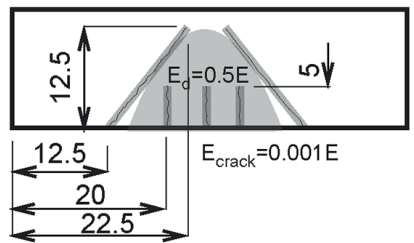

Fig. 4. Degradation models for considered reinforced concrete specimen: a) degradation $\# 1$ - uniformly reduced Young's modulus $E_{d}=0.5 E$ within the parabolic area; b) degradation \#2 - (sub)volume distributed reduced Young's modulus according to proposed spatial function; c) degradation \#3 - reduced Young's modulus $E_{\text {crack }}=0.001 E$ within three vertical and two diagonal cracks; d) degradation \#4 - uniformly reduced Young's modulus $E_{d}=0.5 E$ within the parabolic area (degradation \#1) and reduced Young's modulus $E_{\text {crack }}=0.001 E$ within three vertical and two diagonal cracks (degradation \#3)

as a result of application of a concentrated force at its centre. The distance between supports was $40 \mathrm{~cm}$. Figure 3 shows the front side of the specimen at the last stage of damage (for the force equal to $50 \mathrm{kN}$ ), when damage cracks had length up to $5 / 6$ of the specimen height. To approximate the cracked area the parabola was assigned described with the equation $y(x)=-10.3306 x^{2}+12.5$.

Motivated by the experimental results, four different damage scenarios (Fig. 4) were proposed in this study to reflect the real behaviour of bended beams. As discussed in Section 2.2, all these scenarios were modelled in the FEM formulation by assigning appropriate values of the Young's modulus at selected nodes of the mesh. In the first damage scenario (degradation \#1), the uniformly reduced Young's modulus $E_{d}=0.5 E$ within the parabolic area was introduced. Such damage may be treated as a rough approximation of the micro-cracked zone developed inside a concrete specimen. Degradation \#2 was realized as the parabolic volume with the reduced Young's modulus according to a proposed spatial function (see Fig. 5). The function is re-

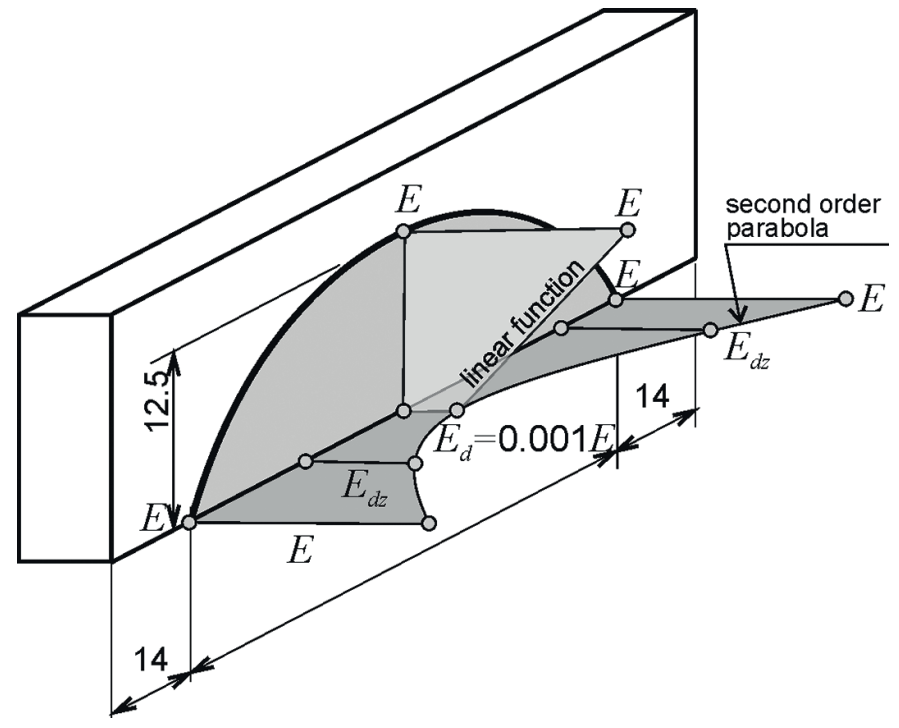

Fig. 5. Geometry of the parabolic volume of degradation 
lated to the area presented in Fig. 3. It is assumed that within the area the Young's modulus value varies in the vertical direction starting from $0.001 E$ at the bottom to $E$ on the top of the parabola. At the same time the function describes the changes of $E$ along the beam axis according to the assumed $2^{\text {nd }}$ order parabola designating the following values: $E, 0.001 E$ and $E$ at spatial positions denoted in Fig. 5. The resulting spatial distribution of $E$ is shown in Fig. 4b as the contour map. Degradation \#3 was considered as an approximation of macro-cracks (i.e. surface-breaking cracks) damage pattern, consisting of three vertical and two diagonal cracks with reduced Young's modulus $E_{\text {crack }}=0.001 E$. The last damage scenario (degradation \#4) was composed as a sum of degradation \#1 and degradation \#3.

3.4. Parameters of wave propagation testing. The excitation signal (Fig. 6) was chosen as a wave packet composed of a fourcycle sinusoidal wave with a central frequency of $96 \mathrm{kHz}$ as a compromise between acceptable level of resolution in the time and frequency domains. The wave packet was modulated by the Hanning window, because the application of the window function results in the excitation of the coherent single-frequency wave packet with significantly reduced side lobes around the carrier frequency.
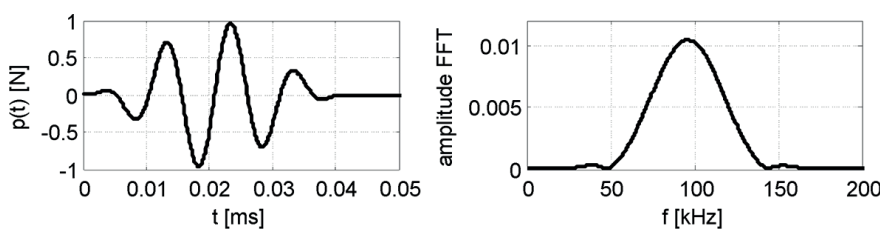

Fig. 6. Excitation signal in time and frequency domain

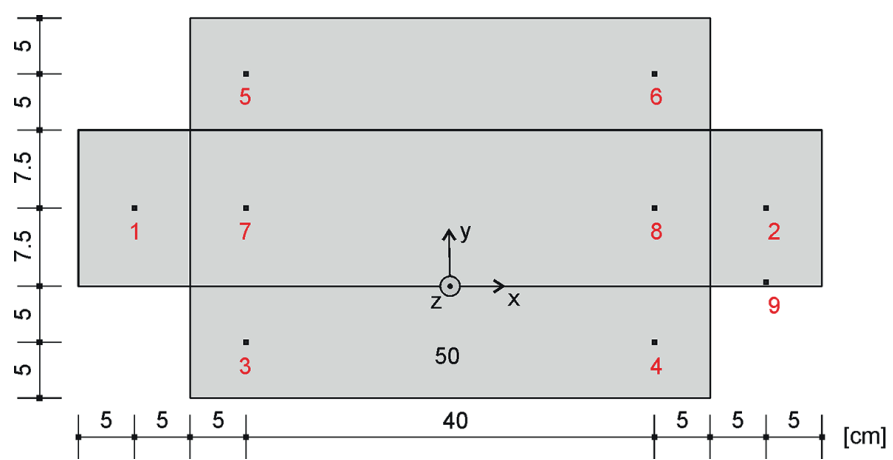

Fig. 7. Location of excitation and registration points on the surface development of the specimen

Figure 7 shows location of excitation and registration points on the surface development of the specimen. The single nodal load was applied perpendicular to the beam surface at node 1 . Acceleration signals were registered at nodes no. 2 to 9 , in the direction perpendicular to the considered surface.

3.5. Analysis of wave propagation results. Acceleration signals registered at points of numbers 2 to 9 for reinforced concrete specimens with various models of degradation are compared in Fig. 8. It can be seen that the degradation has a strong influence on wave propagation signals, especially registered in the area after degradation zone (points of numbers 2, 4, 6, 8, 9), where the largest differences in amplitudes and time-of-flights can be observed. The snapshots of wave propagation patterns for reinforced concrete specimens with various damage scenarios are
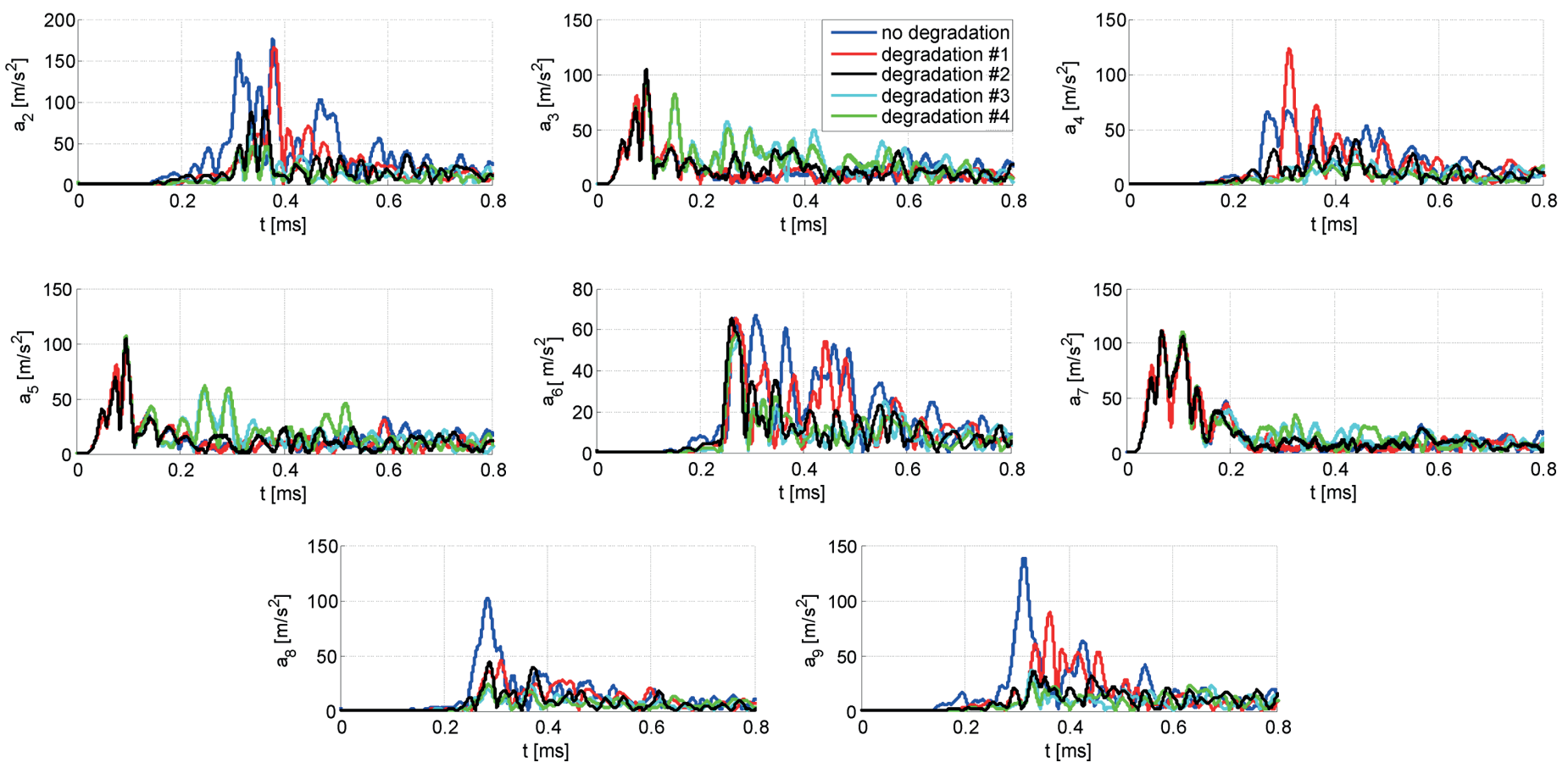

Fig. 8. Envelopes of numerical wave propagation time signals registered at points of numbers 2 to 9 for considered degradation models 

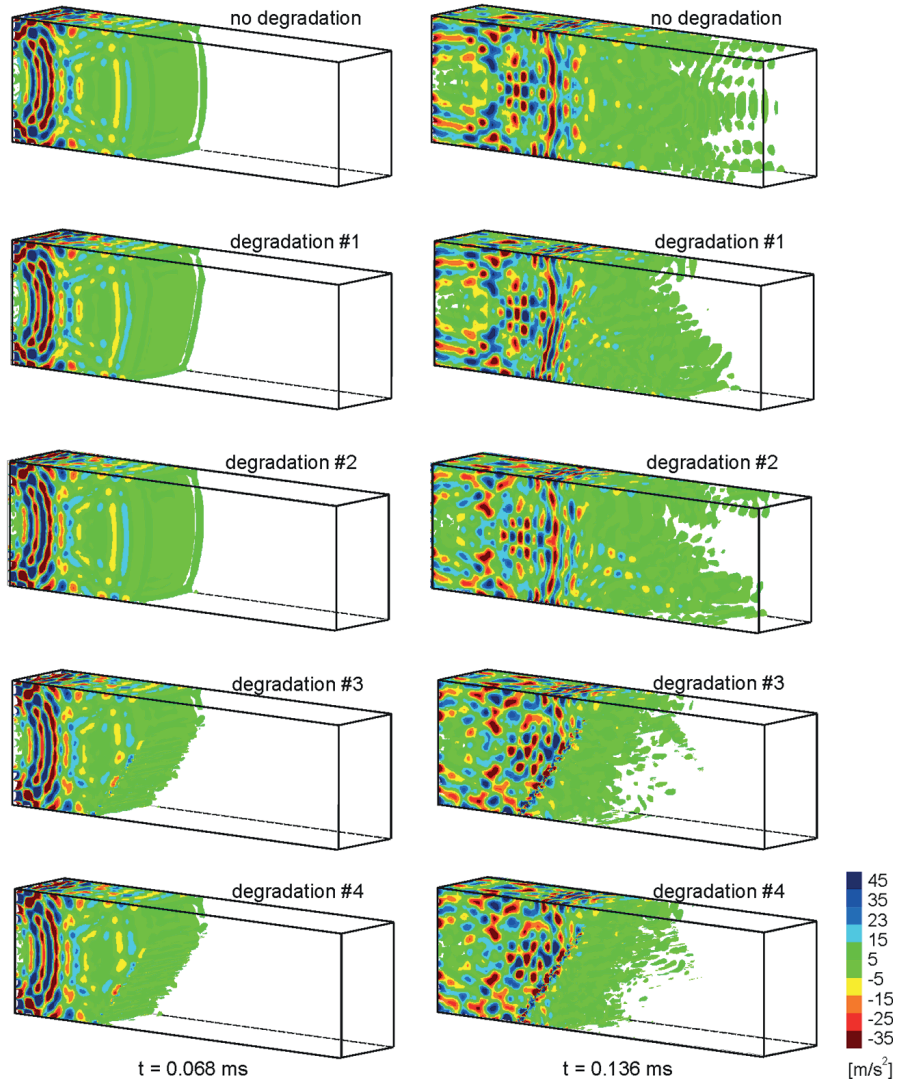

Fig. 9. Wave propagation patterns with reinforced concrete specimens with various damage scenarios at selected time instances $t=0.068 \mathrm{~ms}$ and $t=0.136 \mathrm{~ms}$

shown in Fig. 9. The first selected time instance $(t=0.068 \mathrm{~ms})$ is related to the moment of the arrival of the P-wave front to the half of the specimen span. It can be observed that for various damage scenarios different shapes of the wave front was formulated. At the second time instance $(t=0.136 \mathrm{~ms})$ the P-wave front arrived at the right end of the specimens and the combination of S-waves and R-waves arrived at the degraded zone.

Figure 10 presents experimental signals registered during bending the reinforced specimen described in [11]. The examples of ultrasonic signals acquired at point 2 are given at selected stages of the degradation process (Fig. 11). At stage 1 (Fig. 11a) a single vertical crack of a length approximately $1 \mathrm{~cm}$ is visible. Stage 2 (Fig. 11b) refers to four cracks with the lengths up to

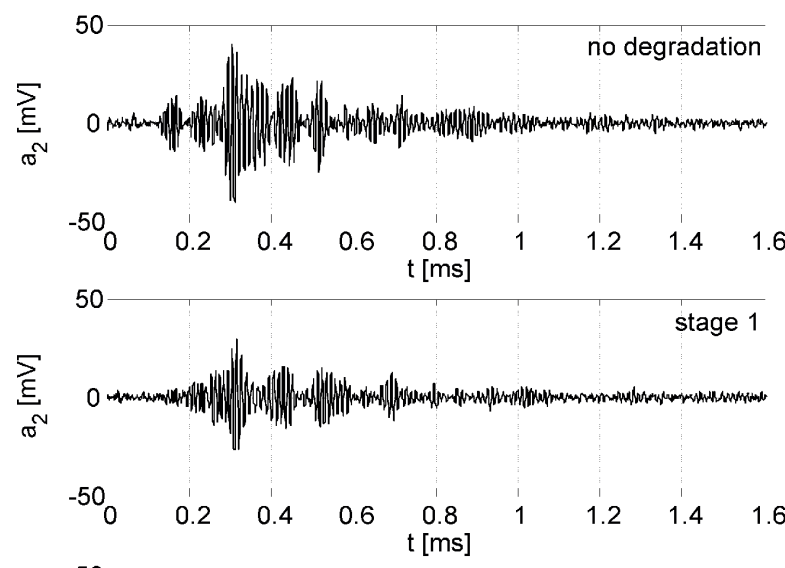

stage 2

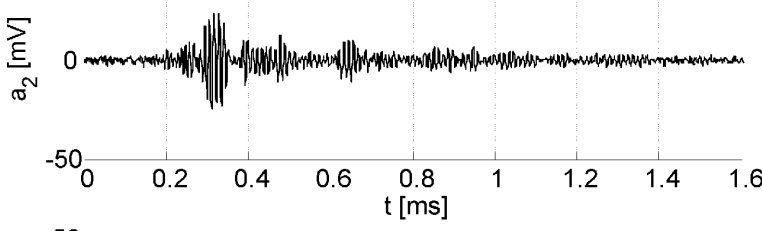

stage 3

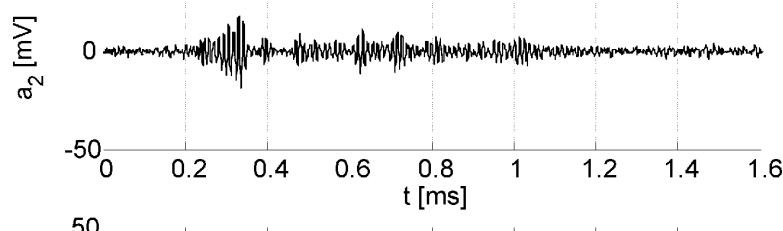

stage 4

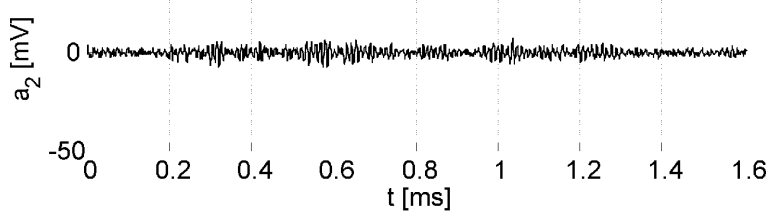

Fig. 10. Experimental wave propagation time signals registered at point 2 [11] for intact specimen and specimens at different stages of degradation: stage 1 (Fig. 11a), stage 2 (Fig. 11b), stage 3 (Fig. 11c), stage 4 (Fig. 11d)

$5 \mathrm{~cm}$. Stage 3 (Fig. 11c) refers to four cracks with the lengths up to $9 \mathrm{~cm}$. The stage 4 (Fig. 11d) corresponds to the cracked specimen (for the force equal to $50 \mathrm{kN}$ ). Figure 10 clearly shows that that after cracking, the amplitude of time signals decreased with the progressive stage of damage. (a)

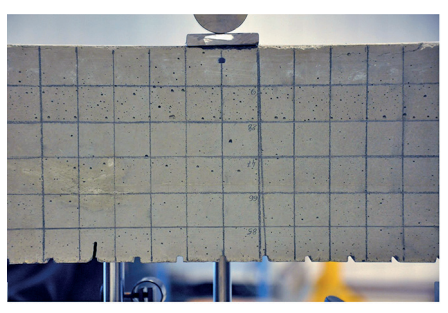

(b)

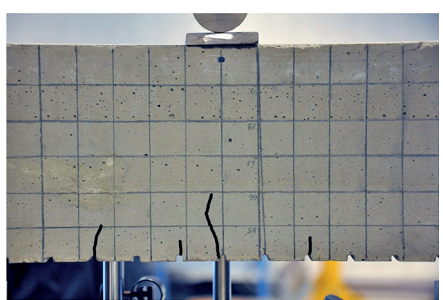

(c)

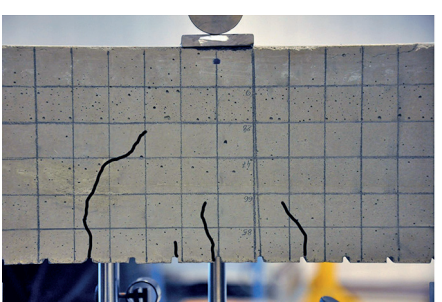

(d)

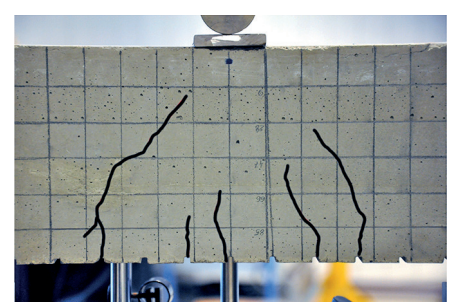

Fig. 11. Crack patterns for RC specimen \#1 subjected to mechanical degradation [11]: a) stage 1; b) stage 2; c) stage 3; d) stage 4 

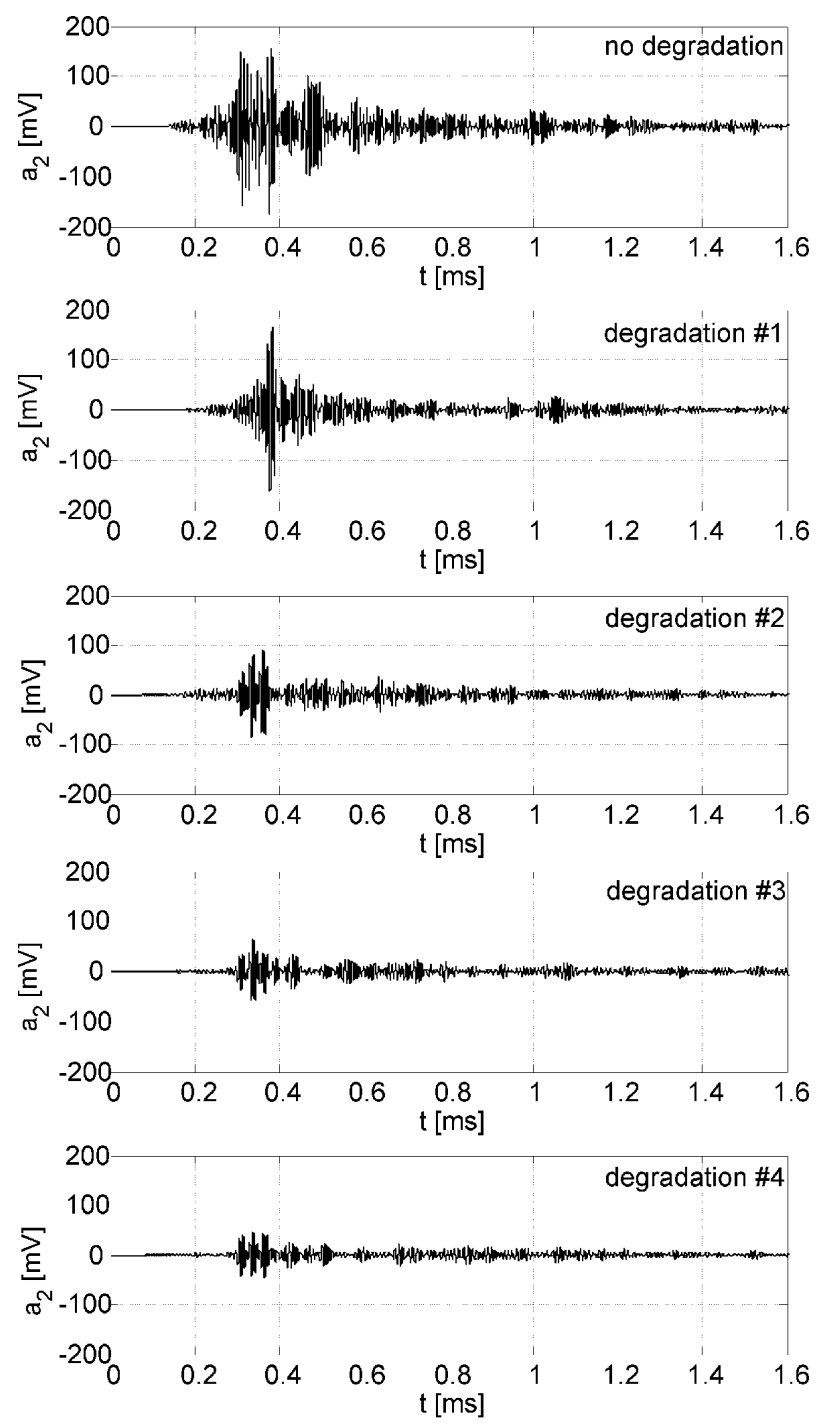

Fig. 12. Numerical wave propagation time signals registered at point 2 for reinforced concrete specimens with various damage scenarios

Numerical signals calculated in Section 2 are illustrated in Fig. 12. As it can be judged from Fig. 10 and Fig. 12, the obtained numerical response corresponds well with the experimental signal, in the undamaged beam. At the beginning of degradation process, only the micro-crack zone appears. The behaviour for this stage can be modelled using the first damage scenario (degradation \#1), with the uniformly reduced Young's modulus within the parabolic area. Later, once some of the micro-cracks will grow to form some major cracks, there exist the volume of material occupied by both microand developing macro-cracks. At this stage damage can be modelled with the use of degradation \#2, for which the value of the Young's modulus along the bottom surface of the beam was significantly reduced, even though this particular model does not take into account "strong" discontinuity in the form of rows of nodes with a reduced value of the Young's modulus. The numerical models with vertical and diagonal cracks (degradation\#3 and degradation\#4) play important role in model- ling of wave propagation in specimens with macro-cracks of significant sizes.

\section{Conclusions}

The paper presents a novel formulation of a 3D spectral element for wave propagation in reinforced concrete. Within the formalism of the spectral element method, the concept of the spatial 3D discretization was proposed. The reinforcement modelled as the truss spectral element was embedded in the 3D solid spectral finite element. Both elements have equal number of nodes in the reinforcement direction and have the same number of degrees of freedom per each node. It was assumed that there exists kinematical consistency between nodal displacements of the truss element and the solid element. This simplifying assumption is justified by a linear character of wave propagation however makes it impossible to diagnose material nonlinearities such for instance debonding between steel and concrete.

The developed 3D spectral element was applied for modelling of wave propagation in reinforced concrete subjected to mechanical degradation. Different degradation models were considered to reflect the real behaviour of bended beams. It was shown that the models incorporating the uniform reduction of the beam stiffness worked well at the early stage of the degradation. The model with the distributed reduction of the stiffness enables to reflect the behaviour of wave propagation at the stage, when macro-cracks are being developed. This model supports the possibility of the use of various spatial functions to analyse different stages of degradation. The formation of physical macro-cracks of large sizes in the more advanced phase of the bending process requires appropriate representation in the numerical model in the form of rows of nodes with a significantly reduced value of the Young's modulus.

The presented research has a preliminary character. The results of the paper may be treated as pilot activities requiring further work in this regard.

Acknowledgements. The research presented in this paper was partially based on the results obtained within the project POIG 01.01.02-10-106/09 "Innovative means and effective methods to improve the safety and durability of building structures and transportation infrastructures in the strategy of sustainable development" (2010-2014), co-financed from EU resources.

\section{REFERENCES}

[1] V. Srinivas, S. Sasmal, K. Ramanjaneyulu, and C. Antony Jeyasehar, "Influence of test conditions on modal characteristics of reinforced concrete structures under different damage scenarios", Archives of Civil and Mechanical Engineering 13, 491-505 (2013).

[2] V. Pérez-Gracia and F. García García, I. Rodriguez Abad, „GPR evaluation of the damage found in the reinforced concrete base of a block of flats: A case study", NDT\&E International 41, 341-353 (2008).

[3] K. Ohno and M. Ohtsu, "Crack classification in concrete based on acoustic emission", Construction and Building Materials 24, 2339-2346 (2010) 
[4] C.-C. Cheng, T.-M. Cheng, and C.-H. Chiang, "Defect detection of concrete structures using both infrared thermography and elastic waves", Automation in Construction 18, 87-92 (2008).

[5] D.G. Aggelis and T. Shiotani, "Repair evaluation of concrete cracks using surface and through-transmission wave measurements", Cement \& Concrete Composites 29, 700-711 (2007).

[6] J. Hoła, Ł. Sadowski, and K. Schabowicz, „Nondestructive identification of delaminations in concrete floor toppings with acoustic methods", Automation in Construction 20, 799-807 (2011).

[7] Y. Yang, G. Cascante, and M.A. Polak, "Depth detection of surface-breathing crack in concrete plates using fundamental Lamb modes", NDT\& E International 42, 501-512 (2009).

[8] J. Hoła, J. Bień, Ł. Sadowski, and K. Schabowicz, „Non-destructive and semi-destructive diagnostics of concrete structures in assessment of their durability", Bulletin of the Polish Academy of Sciences Technical Sciences 63, 87-96 (2015).

[9] A. Garbacz, "Application of stress based NDT methods for concrete repair bond quality control", Bulletin of the Polish Academy of Sciences Technical Sciences 63, 77-85 (2015).

[10] M. Rucka and K. Wilde, "Experimental study on ultrasonic monitoring of splitting failure in reinforced concrete", Journal of Nondestructive Evaluation 32, 372-383 (2013).

[11] M. Rucka and K. Wilde, "Ultrasound monitoring for evaluation of damage in reinforced concrete", Bulletin of the Polish Academy of Sciences: Technical Sciences 63, 1-11 (2015).

[12] B.S. Divsholi and Y. Yang, "Combined embedded and surface-bonded piezoelectric transducers for monitoring of concrete structures", NDT\&E International 65, 28-34 (2014).

[13] F. Moradi-Marani, P. Rivard, C.-P. Lamarche, and S.A. Kodjo, "Evaluating the damage in reinforced concrete slabs under bending test with the energy of ultrasonic waves", Construction and Building Materials 73, 663-673 (2014).

[14] M. Rucka. "Experimental and numerical studies of guided wave damage detection in bars with structural discontinuities", Archive of Applied Mechanics 80, 1371-1390 (2010).

[15] M. Rucka. "Modelling of in-plane wave propagation in a plate using spectral element method and Kane-Mindlin theory with application to damage detection", Archive of Applied Mechanics 81, 1877-1888 (2011).

[16] T. Patera, "A spectral element method for fluid dynamics: laminar flow in a channel expansion", Journal of Computational Physics 54, 468-488 (1984).

[17] J. F. Semblat and J. J. Brioist, "Efficiency of higher order finite elements for the analysis of seismic wave propagation", Journal of Sound and Vibration 231, 460-467 (2000).

[18] D. Komatitsch, R. Martin, J. Tromp, M.A. Taylor, and B.A. Wingate, "Wave propagation in 2-D elastic media using a spectral element method with triangles and quadrangles", Journal of Computational Acoustics 9, 703-718 (2001).

[19] W. Ostachowicz, P. Kudela, M. Krawczuk, and A. Żak Guided Waves in Structures for SHM: The Time-Domain Spectral Element Method, Wiley, 2012.

[20] A. Żak, M. Krawczuk, and W. Ostachowicz, „Propagation of inplane wave in an isotropic panel with a crack", Finite Elements in Analysis and Design 42, 929-941 (2006).
[21] M. Rucka, W. Witkowski, J. Chróscielewski, and K. Wilde, "Damage detection of a T-shaped panel by wave propagation analysis in the plane stress", Archives of Civil Engineering LVIII(1), 3-24 (2012).

[22] J. Chróścielewski, M. Rucka, W. Witkowski, and K. Wilde, "Formulation of spectral truss element for guided waves damage detection in spatial steel trusses", Archives of Civil Engineering LV(1), 43-63 (2009).

[23] A. Żak, "A novel formulation of a spectral plate element for wave propagation in isotropic structures", Finite Elements in Analysis and Design 45, 650-658 (2009).

[24] Y. Liu, N.Hu, C.Yan, X.Peng, and B.Yan, "Construction of a Mindlin pseudospectral plate element and evaluating efficiency of the element", Finite Elements in Analysis and Design 45, 538-546 (2009).

[25] W. Witkowski, M. Rucka, J. Chróścielewski, and K. Wilde, "Wave propagation analysis in spatial frames using spectral Timoshenko beam elements in the context of damage detection". Archives of Civil Engineering LV(3), 367-402 (2009).

[26] A. Żak and M. Krawczuk, "Certain numerical issues of wave propagation modelling in rods by the Spectral Finite Element Method", Finite Elements in Analysis and Design 47, 1036-1046 (2011).

[27] L. Ge, X. Wang, and C. Jin, "Numerical modelling of PZT-induced Lamb wave-based crack detection in plate-like structures", Wave Motion 51, 867-885 (2014).

[28] H. Peng, Meng G., and F. Li, "Modeling of wave propagation in plate structures using three-dimensional spectral element method for damage detection", Journal of Sound and Vibration 320, 942-954 (2009).

[29] A. Żak, M. Krawczuk, Ł. Skarbek, and M. Palacz, "Numerical analysis of elastic wave propagation in unbounded structures", Finite Elements in Analysis and Design 90, 1-10 (2014).

[30] D.M. Joglekar and M. Mitra, "Nonlinear analysis of flexural wave propagation through 1D waveguides with breathing crack", Journal of Sound and Vibration 344, 242-257 (2015).

[31] R. Sridhar, A. Chakraborty, and S. Gopalakrishnan, "Wave propagation analysis in anisotropic and inhomogeneous uncracked and cracked structures using pseudospectral finite element method", International Journal of Solids and Structures 43, 4997-5031 (2006).

[32] O.C. Zienkiewicz and R.L. Taylor, The Finite Element Method, Butterworth-Heinemann, 2000.

[33] T.J.R. Hughes, The Finite Element Method: linear static and dynamics finite element analysis, Dover Publications, Inc., New York, 2000.

[34] W. Witkowski, M. Rucka, J. Chróścielewski, and K. Wilde, "On some properties of 2D spectral finite elements in problems of wave propagation", Finite Elements in Analysis and Design 55, 31-41 (2012)

[35] M. Koizumi, "FGM activities in Japan", Composites Part B 28B, 1-4 (1997).

[36] J. Woo and S. A. Meguid, "Nonlinear analysis of functionally graded plates and shallow shells", International Journal of Solids and Structures 38, 7409-7421, (2001). 